\title{
Hydrodynamics of Young Binaries with Low-Mass Secondaries
}

\author{
Tatiana Demidova ${ }^{1}$, Vladimir Grinin ${ }^{1,2}$ and Nataliya Sotnikova ${ }^{2}$ \\ ${ }^{1}$ Pulkovo Astronomical Observatory of the Russian Academy of Sciences, \\ 196140, Pulkovskoye chaussee 65/1, Saint-Petersburg, Russia \\ email: proxima10list.ru \\ ${ }^{2}$ Saint-Petersburg State University, V.V. Sobolev Astronomical Institute, \\ 198504, Universitetskij pr. 28, Petrodvorets, Saint-Petersburg, Russia
}

\begin{abstract}
The model of a young star with a low-mass secondary component $\left(q=M_{2} / M_{1} \leqslant\right.$ 0.1 ) accreting matter from a circumbinary $(\mathrm{CB})$ disc is considered. It is assumed that the orbit and the $\mathrm{CB}$ disc can be coplanar and non-coplanar. The model parameters were varied within the following ranges: the component mass ratio $q$ ranged from 0.1 to 0.003 , the eccentricity $e$ varied from 0 to 0.7 , the inclination of the orbit plane to the $\mathrm{CB}$ disc ranged from 0 to 10 degrees, and the parameter that defines the viscosity of the system was also varied. A number of hydrodynamics models of such a system have been calculated by the SPH method and then the variations of the circumstellar extinction and phase brightness curves were determined. The calculated brightness curves differ in shape and amplitude and it depends on the model parameters and the orientation of the system relative to the observer. The results were used to analyze the cyclic activity of UX Ori type stars.
\end{abstract}

Keywords. accretion, accretion discs, hydrodynamics, numerical method, close binaries

\section{Introduction}

The variable circumstellar extinction is one of the reasons for the photometric activity of young stars. This variability can be easily recognized in the UX Ori type stars because of the "optimal" orientation of their circumstellar discs with respect to the line of sight (Grinin et al. 1991). The photometric observations of these stars show that there are cyclic large-scale brightness variations along with chaotic minima of brightness (Schevchenko et al. 1993, Grinin et al. 1998, Rostopchina 1999). The cyclic variability reflects the existence of stable dust structures around the stars. Grinin et al. (1998) and Bertout (2000) suggested that such structures could be density waves and streams of matter caused by the motion of a small companion or a giant planet. This was first predicted by Artymowicz \& Lubow (1996). We considered models of a young binary system accreting matter from a circumbinary disc. It is shown that tidal forces create a gap in the innermost part of a disc. Under certain circumstances, two unequal streams of matter penetrate the gap due to viscosity forces and gravitational perturbations. They feed the accretion discs around the binary components. Artymowicz \& Lubow (1996) showed that the accretion rate in an eccentric binary varies strongly in time and depends strongly on the phase of the orbital period.

What would be the behavior of such a system if it was observed edge-on or at a slight angle to the line of sight? For the first time, this question was discussed in the paper Sotnikova \& Grinin (2007). These authors showed that the column density of matter along the line of sight can be a complicated function of the phase of the orbital period. Similar calculations have been continued in our subsequent papers (Demidova et al. 
2010, Grinin et al. 2010a). We considered two types of binary orbits. In the first case, an orbit was chosen coplanar with a circumbinary disc plane. In the second one, it was inclined at a small angle to the disc plane. The latter case was investigated earlier by Mouillet et al. (1997) and Larwood \& Papaloizou (1997). The authors have modelled the circumstellar disc of $\beta$ Pic, which has an inner part inclined by a few degrees with respect to the outer one (Burrows et al. 1995). It was shown that the inclination was caused by the motion of a planet on an orbit inclined at a small angle to the circumstellar disc. Recently, the same situation was observed in the circumstellar disc of CQ Tau star (Eisner 2004, Chapillon 2008). Both these events allow us to suggest that such situations are not so rare and more detailed calculations are needed. In this paper, we model the brightness curves of binary systems, orbiting at a slight angle to the circumbinary disc plane.

\section{Overview}

We used the SPH method to calculate hydrodynamic flows in a series of models of young binaries embedded into a gas-dust disc. The column density of matter in the direction to the line of sight was calculated in the course of simulations. It turned out that it depends on the inclination of the $\mathrm{CB}$ disc to the line of sight $(\phi)$ and the position of the apsidal line relative to an observer $(\theta)$. Input parameters were: the component mass ratio - $q=0.1-0.003$, eccentricity - $e=0-0.7$, the period of the binary $-P=1-5$ years, parameter $c$ (the speed of sound), which characterizes the turbulent viscosity parameter, the inclination of the binary orbit to the circumbinary disc plane - $\psi$, the accretion rate $\dot{M}_{a}$, and opacity per gram of matter $-\kappa$. The dust-to-gas ratio was taken to be 1:100, close to the value in the interstellar medium. The computations were performed for a few hundred orbital periods. The number of SPH particles involved in the simulations ranged from 60,000 to 200,000. We used the input parameters to transform the column density of SPH particles to optical depth. Then, we calculated the theoretical brightness curves (see Demidova et al. (2010) for more details).

The calculations show that the brightness variations with the orbital period can be observed even if the mass of the companion is one hundredth of the mass of the primary star. The amplitudes of variations ranged from 0.8 to 2 magnitudes in the models with the mass ratio $q=0.01$ and $q=0.003$, provided moderate values of accretion rate $\left(\dot{M}_{a}=10^{-9} M_{\odot} / y r\right)$.

For the eccentric binaries, the form and amplitude of brightness variations strongly depend on the orientation of the system with respect to an observer. The same effect arises in the case of circular orbits if the binary's orbital plane is misaligned with the plane of the CB disc. Relatively small changes in the system orientation lead to considerable changes of the brightness curve form. Depending on the position of the line of sight, two types of cyclic activity are possible. The first one is described by two-component brightness curves (see Fig. 1). A prolonged eclipse is caused by the extinction in the stream of matter, moving to the primary component. The second, more compact, eclipse is due to extinction in the denser stream of matter directed to the secondary component. Cyclic activity of the second type demonstrates the simple almost sinusoidal brightness curve (Fig. 2). In this case, there is only one stream of matter, which moves to the primary component and crosses the line of sight, provided an appropriate orientation of the system. Our analysis shows that the first type of activity is more common for the misaligned models. The latter one is observed if the binary's orbital plane is coplanar with the CB disc plane. 


\section{Implications}

We compared the observed brightness curves with theoretical ones and found many common features. For example, some of UX Ori stars show two-component cycles. Fig. 1 demonstrates such a case for BF Ori (Grinin et al. 2010b). We obtained a similar brightness curve for the simple model of the binary with the CB disc inclined at small angle to the line of sight.

However, most of UX Ori type stars demonstrate the simple cycles, for instance CO Ori (Rostopchina 2007). It should be noted that the simple brightness curves can be obtained in models with noticeably different parameters. Therefore, we need some additional information on the system parameters (the inclination of the disc plane to the line of sight, radial velocity variations, etc.) to chose the "best fit model".

Unfortunately, the photometric cycles of UX Ori type stars are not studied well enough because the cycle periods are rather long, but the series of observations, in contrast, are short. Nevertheless, our calculations show that at least photometric cycles of some UX Ori stars can be caused by the binarity.

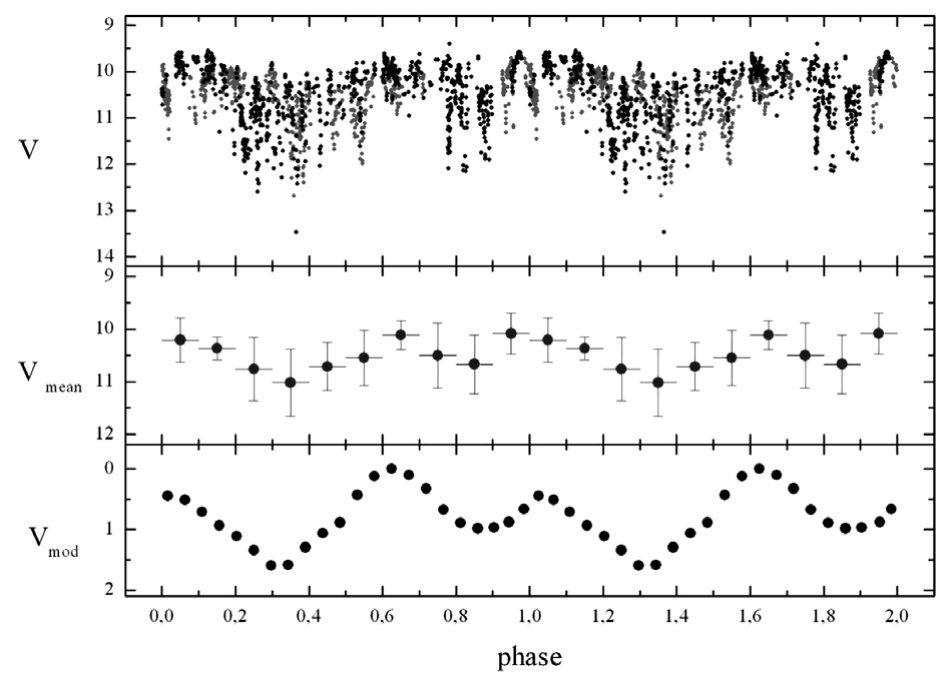

Figure 1. Top and center are the brightness curve of BF Ori, folded with period 12 yr. Bottom is the model brightness curve: $c=0.05, q=0.01, e=0.5, \psi=5^{\circ}, \theta=180^{\circ}, \phi=7^{\circ} .5$, $\dot{M}_{\mathrm{a}}=10^{-9} M_{\odot} / \mathrm{yr}$.
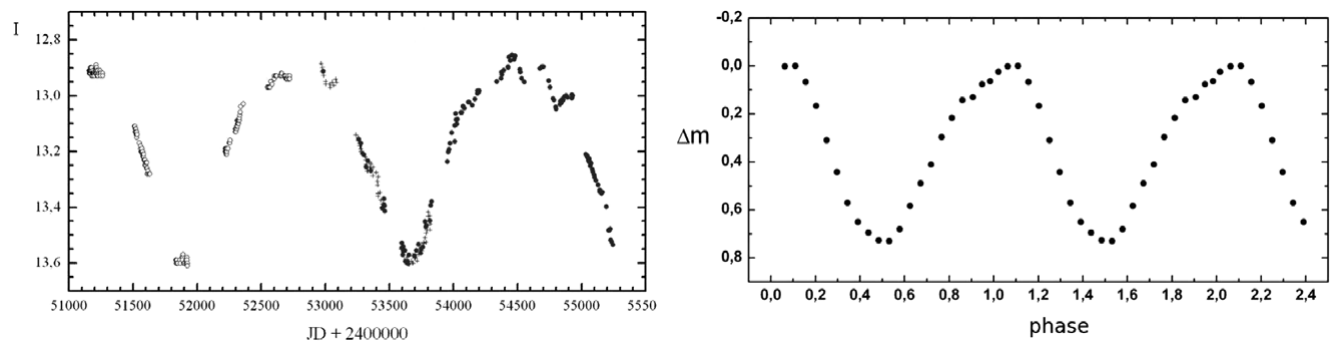

Figure 2. Left is the brightness curve of V718 Per. Right is the brightness curve of the model: $c=0.05, M_{2}=6 M_{\mathrm{J}}, e=0.0, \psi=10^{\circ}, \theta=45^{\circ}, \phi=7^{\circ}, \dot{M}_{\mathrm{a}}=2 \cdot 10^{-10} M_{\odot} / \mathrm{yr}$.

The results of our simulations can also be applied to young stars with long-lasting eclipses. One of such stars is the weak-lined T Tauri star V718 Per. The duration of its 
eclipses is about 3.5 yrs and the period is about 4.7 yrs. The star does not show the presence of the secondary component within the errors of the observations: according to (Grinin et al. 2008), the mass of an expected planet has to be less than 6 Jupiter masses. We obtained a similar brightness curve in a model with the mass of secondary about 6 Jupiter masses (Fig. 2). Thus, the discussed models qualitatively describe the cyclic activity of UX Ori type stars and can be used to investigate young stars with abnormal prolonged eclipses.

\section{References}

Artymowicz, P. \& Lubow, S. H. 1996, ApJ, 467, L77

Bertout, C. 2000, A\&GA, 363, 984

Burrows, C. J., Krist, J. E., Stapelfeldt, K. R. \& WFPC2 Investigation Definition Team 1995, $B A A S, 27,1329$

Chapillon, E., Guilloteau, S., Dutrey, A., \& Pie'tu, V. 2008, A\&A, 488, 565

Demidova, T. V., Grinin, V. P., \& Sotnikova, N. Ya. 2010, Pisma Astron. Zh., 36, 526 [Astron. Lett., 36, 498 (2010)]

Eisner, J. A., Lane, B. F., Hillenbrand, L. A., Akeson, R. L., \& Sargent, A. I. 2004, ApJ, 613, 1049

Grinin, V. P., Kiselev, N. N., Minikulov, N. Kh. et al. 1991, ApESSS, 186, 283

Grinin, V. P., Rostopchina, A. N., \& Shakhovskoi, D. N. 1998, Pisma Astron. Zh., 24, 925 [Astron. Lett., 24, 802 (1998)]

Grinin, V. P., Stempels, E., Gahm, G. et al. 2008, A\&A, 489, 1233

Grinin, V. P., Demidova, T. V., \& Sotnikova, N. Ya., 2010a, Pisma Astron. Zh., 36, 584 [Astron. Lett., 36, 808 (2010)]

Grinin, V. P., Rostopchina, A. N., Barsunova, O. U., \& Demidova, T. V. 2010b, Astrophysics 53,367

Larwood, J. D. \& Papaloizou, J. C. P. 1997, MNRAS, 285, 288

Mouillet, D., Larwood, J. D., Papaloizou, J. C. B., \& Larange, A. M. 1997, MNRAS, 292, 896

Rostopchina, A. N. 1999, Astron. Zh., 76, 136 [Astron. Rep., 43, 113 (1999)]

Rostopchina, A. N., Shakhovskoi, D. N, Grinin, V. P., \& Lomach, A. A. 2007, Astron. Zh., 84, 60 [Astron. Rep., 51, 55 (2007)]

Sotnikova, N. Ya. \& Grinin, V. P. 2007, Pis'ma Astron. Zh., 33, 667 [Astron. Lett., 33, 594 (2007)]

Schevchenko, V. S., Grankin, K. N, Ibragimov, M. A. et al. 1993, ApESSS, 202, 137

\section{Discussion}

R. BALUEV: Is it more likely to form a planet around a binary or a single star?

T. Demidova: We did not investigate this question.

M. Montgomery: How did you get your inner disk to tilt and by how much?

T. Demidova: The tilt is an initial condition and ranges from 1 to 10 degrees.

A. Triaud: Can you make an inner disc perpendicular compared to the star or outer disc by placing a planet on a higher obliquity?

T. Demidova: This is a preliminary result. The small obliquity was to show how viscosity affects the disc. Higher obliquities have not been tried. 